\title{
Gain-Scheduling Multivariable LPV Control of an Irrigation Canal System
}

\author{
Yolanda Bolea, Vicenç Puig \\ Advanced Control Systems Group (SAC) \\ IRI Institut de Robòtica i Informàtica Industrial (CSIC-UPC) \\ Universitat Politècnica de Catalunya (UPC) \\ Pau Gargallo, 5, 08028 Barcelona, Spain
}

\begin{abstract}
The purpose of this paper is to present a multivariable linear parameter varying (LPV) controller with a gain scheduling Smith Predictor (SP) scheme applicable to open-flow canal systems. This LPV controller based on SP is designed taking into account the uncertainty in the estimation of delay and the variation of plant parameters according to the operating point. This new methodology can be applied to a class of delay systems that can be represented by a set of models that can be factorized into a rational multivariable model in series with left/right diagonal (multiple) delays, as, e.g. the case of irrigation canals. A multiple pool canal system is used to test and validate the proposed control approach.
\end{abstract}

Keywords: Linear Parameter Varying Control, Gain scheduling, Open-flow canals.

\section{INTRODUCTION}

Distributed parameter systems (Belforte et al., 2002), usually considered as models with a very large number of states could be approximated with low order linear time invariant (LTI) models in order to use classical linear control design tools, as it is usual in control engineering practice. However, simplified LTI parameter models lose all information about the spatial structure of the original system and cannot account for it, although they are satisfactory from an input-output point of view. Hence, simplified model structures that still preserve the information about the non-linearity, the influence of the operating point and the system spatial structure are needed to design a satisfactory control. Such structure can be provided by linear parameter varying (LPV) models consisting of a linear lumped model in which the system parameters are function of external measurements, or by quasi-LPV models 
when the parameters vary according to states and/or operating conditions of the system (Rugh and Shamma, 2000). LPV models (Bamieh and Giarre, 2002 ) (De Caigny et al., 2009 ) are suitable for gainscheduling (GS) control design. Conventional GS techniques have been widely used for designing controllers for multiple industrial applications (where the system parameters are varying) (see, e.g. Leith and Leithead, 2000). The conventional GS is a heuristic method (Shamma, 1991) that consists in dividing the parameter space into small regions (operating points), in which the plant is regarded as a LTI system. Then, LTI controllers are designed for each operating point to achieve a synthetic controller with the use of interpolation. The heuristic gain scheduled controllers normally guarantee control system stability when the parameters vary slowly, although sometimes it may lead to instability or chaotic behavior. In fact, the main known drawback of this technique is that it does not guarantee stability and performance rigorously and it does not provide a systematic design procedure. On the other hand, LPV GS design can be formulated as a convex optimization problem based on linear matrix inequality (LMI) constraints, with stability and performance guarantees (Becker and Packard, 1994) (Apkarian, 1995a) (Apkarian, 1995b). In recent years, a significant numbers of contributions to the LPV theory can be found in the literature (Boudaoud et al., 2014) (Castillo et al., 2015) (Guoyan et al., 2015) (Hwanyub and Sung, 2015) (Liang et al., 2015) (Masubuchi and Kurata, 2011)(Sato and Peaucelle, 2013) (Wang et al., 2015) (White et al., 2013).

The main contribution of this paper is to propose a multivariable control design methodology applicable to open-flow canal systems. Open-flow canals involve mass energy transport phenomena they behave as intrinsically distributed parameter systems that can be approximated by means of LPV as discussed in (Bolea, 2014b). The proposed control approach is based on a LPV GS controller coupled with a delay-scheduling MIMO Smith Predictor that considers model uncertainty extending the results presented in (Sanchez-Peña, 2009) for the LTI case where a robust controller designed from Youla parametrization is used. In the proposed methodology, a set of necessary and sufficient conditions under which a model can be separated into diagonal left and right pure delays and a rational matrix is 
presented. Surprisingly, these conditions are satisfied by multiple pool open flow canal systems. The varying parameters are measured in real time and used to schedule the controller parameters. The "delay scheduling" Smith predictor scheme is used to compensate most of the estimated delay. However, there is still a remaining delay due to the inaccuracy in its estimation that will be represented as unstructured (multiplicative) dynamic uncertainty in a robust control framework. Performance is quantified as an induced $L_{2}$ norm, as part of a mixed sensitivity problem (MSP) with measured varying parameters. This problem can be solved by means of the standard LPV theory using LMI optimization (Becker and Packard, 1994). In order to design the controller, the region of parameter variations is approximated by a polytopic region that reduces the number of LMIs to be solved to a finite number, one for each vertex of the polytopic region (Apkarian, 1995a) (Apkarian, 1995b). To validate the proposed LPV GS controller a two-pool open flow canal system is used.

The structure of the paper is as follows: In Section II, the LPV a description of a multiple pool canal plant is presented. In Sections $I I I$ and $I V$, the formulation, synthesis and implementation of a gainscheduling LPV controller in conjunction with a "delay scheduling" Smith predictor (LPV SP) are presented. In Section $V$, the proposed methodology is applied to a two-pool canal. Conclusions are drawn in Section VI.

\section{MULTIPLE POOL IRRIGATION CANAL SYSTEMS}

An irrigation canal is an open-flow hydraulic system, whose objective is mainly to convey water from reservoirs down to its final users. To achieve this aim, hydraulic gates are operated in order to control the water levels downstream of the pools (Figure 1). In this figure, a simplified view of our canal of study is presented. It receives water of a source, reservoir, and lets the water freely flow by gravity following the slope. The following variables are considered to define the input-output behavior of the canal as a dynamic system: the water levels $y_{d i}$ at the downstream end of each pool are the outputs to be controlled and the gate openings $u_{i}$ are the manipulated control inputs. The overall objective of the 
control scheme is to drive automatically the gate openings to ensure that the downstream levels reach and keep their prescribed set-points in spite of the disturbances.

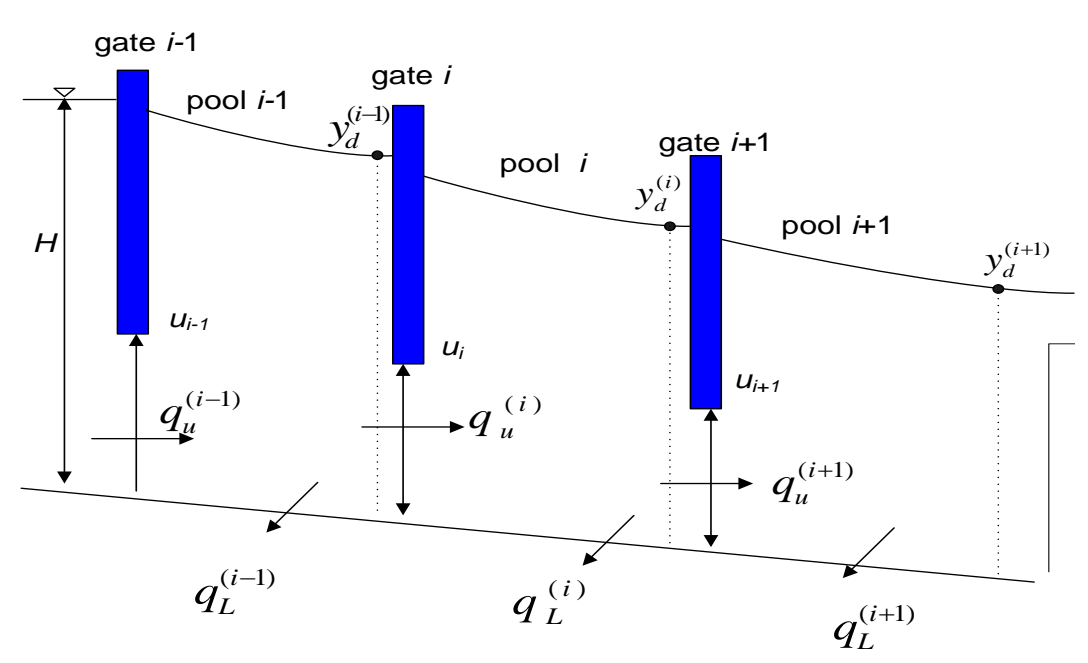

Figure 1. Irrigation canal system

Open-flow canals involve mass energy transport phenomena which behave as intrinsically distributed parameters systems. Their complete dynamics is represented by non-linear partial differential hyperbolic equations, Saint-Venant equations, which have no analytic solution for arbitrary geometry. They express the conservation of mass (1), and momentum principles (2) in a one-dimensional free surface flow (Barré de Saint Venant, 1871) (Chow, 1959):

$$
\begin{gathered}
\frac{\partial q}{\partial x}+\frac{\partial A}{\partial t}=0 \\
\frac{\partial q}{\partial t}+\frac{\partial}{\partial x}\left(\frac{q^{2}}{A}\right)+g A \frac{\partial y}{\partial x}-g A\left(I_{0}-I_{f}\right)=0
\end{gathered}
$$


Here $q=q(x, t)$ is the flow $\left[\mathrm{m}^{3} / \mathrm{s}\right], A=A(x, t)$ is the cross-sectional area $\left[\mathrm{m}^{2}\right], t$ is the time variable $[\mathrm{s}]$, $x$ is the spatial variable $[\mathrm{m}]$, measured in the direction and the sense of the movement, $g$ is the gravity $\left[\mathrm{m} / \mathrm{s}^{2}\right], I_{0}$ is the bottom slope and $I_{f}$ is the friction slope.

However, Saint-Venant equations are not useful for designing a controller using linear theory as already noticed by (Litrico and Fromion, 2006). Instead, a simplified control-oriented model (around a given operating point) is proposed (Bolea et al., 2014b), that extends the IDZ (integral delay zero) model proposed in (Litrico, 2004) to the LPV case, with the aim of designing the control through the proposed methodology explained in detail in the next sections. According to this modeling approach, the single reach canal reach dynamics (relation between upstream and downstream levels, $y_{u}$ and $y_{d}$, and downstream flow, $q_{d}$, and upstream flow, $q_{u}$ ) for low frequencies can be approximated by

$$
\begin{gathered}
y_{u}(s)=P_{11}(s) q_{u}(s)+P_{12}(s) q_{d}(s) \\
y_{d}(s)=P_{21}(s) q_{u}(s)+P_{22}(s) q_{d}(s)
\end{gathered}
$$

where:

$$
P_{11}(s)=\frac{1}{A_{u} s}, \quad P_{12}(s)=\frac{-e^{-\tau_{u} s}}{A_{u} s}, P_{21}(s)=\frac{e^{-\tau_{d} s}}{A_{d} s} \text { and } P_{22}(s)=\frac{-1}{A_{d} s}
$$

with $\tau_{u}$ and $\tau_{d}$ are the upstream and downstream transport delays and $A_{u}$ and $A_{d}$ are the upstream and downstream backwater areas, all depending on the operating point.

\section{MIMO LPV SMITH PREDICTOR}

\section{A. Problem set-up}

Let us consider a time-delayed MIMO LPV system ${ }^{1}$ :

$$
G(s, \theta)=G_{m}(s, \theta) * D(s, \theta)
$$

where ".*” is the element-wise matrix product (Hadamard product) and

\footnotetext{
${ }^{1}$ For simplicity and with abuse of notation, LPV systems are presented as transfer functions in this section, although computations are performed entirely using the state space representation.
} 


$$
\begin{aligned}
& G_{m}(s, \theta)=\left[g_{i j}(s, \theta)\right] \\
& D(s, \theta)=\left[e^{-s \tau_{i j}(\theta)}\right], i=1, \ldots, n ; j=1, \ldots, m
\end{aligned}
$$

The time varying parameter vector $\theta(t)$ can be measured (estimated) in real time and can be used to adapt the controller using a gain-scheduling controller scheme as in (Apkarian, 1995a) (Bolea et al., 2014a). Time delays are compensated using a "delay scheduling" MIMO Smith predictor scheme (Figure 2). However, since there is always an error in the delay estimation (even when the time delay variation with the operating point is modeled), a remaining delay due to the inaccuracy in its estimation still is present. This may be represented as unstructured dynamic uncertainty in a robust control framework, which we assume here as LTI. The uncertain model is obtained from the Smith Predictor structure (Figure 2), between input $u$ and the feedback output $f$ :

$$
\begin{aligned}
& G(s, \theta)(I+\Delta(s))-G(s, \theta)-G_{m}(s, \theta) \\
& =G(s, \theta) \Delta(s)-G_{m}(s, \theta)
\end{aligned}
$$

The model uncertainty in the delay can be covered by the following global dynamic uncertain set of models:

$$
G(s, \theta)=\left\{\left[I+\Delta W_{\delta}(s)\right] G_{m}(s, \theta), \quad \Delta \in C, \bar{\sigma}(\Delta)<1\right\}
$$

with $W_{\delta}(s)=w_{\delta}(s) I$ and $w_{\delta}(s)$ a SISO stable non-minimum phase linear model.

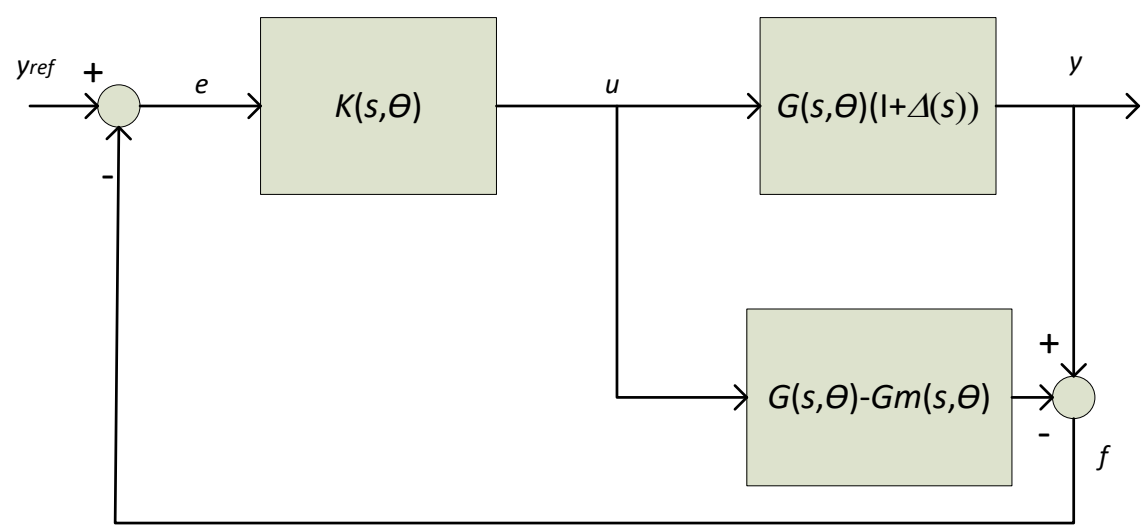

Figure 2. MIMO Smith Predictor 


\section{B. Left/right delay factorization}

In (Sanchez-Peña et al., 2009), it has been proved that a left/right decomposition should be used to obtain an accurate bound of model uncertainty in case of using a MIMO Smith Predictor. Here, the LPV extension of this reasoning is presented:

$$
\begin{aligned}
& G(s, \theta)=\left[g_{i j}(s, \theta) e^{-s \tau_{i j}(\theta)}\right] \\
& =\operatorname{diag}\left[e^{-s \tau_{i}^{l}(\theta)}\right] G_{m}(s, \theta) \operatorname{diag}\left[e^{-s \tau_{j}^{r}(\theta)}\right]
\end{aligned}
$$

To find these left $\left(\tau_{i}^{l}(\theta)\right)$ and right delays $\left(\tau_{i}^{r}(\theta)\right)$, we need to solve the following linear equation:

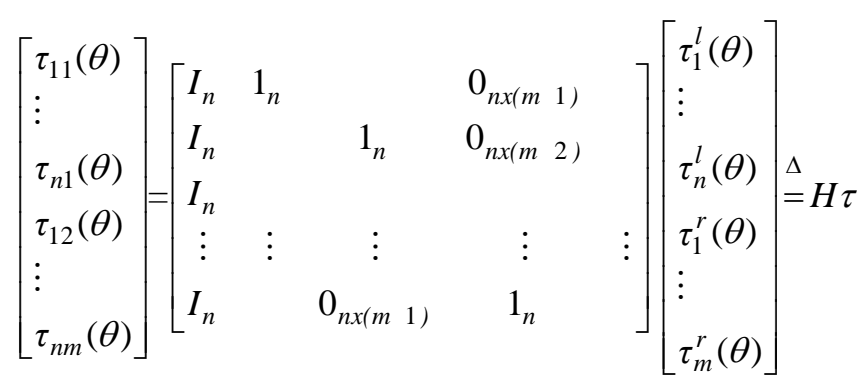

with $H \in \mathfrak{R}^{n m \times(n+m)}$, and $\mathbf{1}_{n}\left(\mathbf{0}_{n}\right)$ a column vector of ones (zeros) of length $n$. According to (Sanchez-Peña et al., 2009) and considering the LPV case, for MIMO systems in general, there exists a solution to the previous linear equation if and only if the following relations hold:

$$
\tau_{i, q}(\theta)-\tau_{j, q}(\theta)=\tau_{i, k}(\theta)-\tau_{j, k}(\theta), \begin{cases}i=1, \ldots, n & j=1, \ldots, n \\ q=1, \ldots, m & k=1, \ldots, m\end{cases}
$$

This seems an artificial condition, but surprisingly, it is satisfied by multiple pool open flow canals, as the ones presented in Section II and Section $V$ by means of an example. For these particular system structures, less restrictive conditions can be stated, as will be proved next.

\section{Delay uncertainty}


Once the factorization of the plant's model which separates delays from rational part has been made, the delay uncertainty is considered. The uncertain model of the plant is:

$$
\begin{aligned}
& G(s, \theta)=D_{l}(s, \theta) G_{m}(s, \theta) D_{r}(s, \theta)=\Delta_{l}(s) G_{o}(s, \theta) \Delta_{r}(s) \\
& D_{l}(s, \theta)=\operatorname{diag}\left[e^{s \tau_{i}^{l}(\theta)}\right]= \\
& =\Delta_{l} \bar{D}_{l}(s, \theta) \stackrel{\Delta}{=} \operatorname{diag}\left[e^{s \delta_{i}^{l}}\right] \operatorname{diag}\left[e^{s \bar{\tau}_{i}^{l}(\theta)}\right], i=1, \ldots n \\
& D_{r}(s, \theta)=\operatorname{diag}\left[e^{s \tau_{j}^{r}(\theta)}\right]= \\
& =\Delta_{r} \bar{D}_{r}(s, \theta) \stackrel{\Delta}{=} \operatorname{diag}\left[e^{s \delta_{j}^{r}}\right] \operatorname{diag}\left[e^{s \bar{\tau}_{j}^{r}(\theta)}\right] j=1, \ldots m \\
& G_{o}(s, \theta) \stackrel{\Delta}{=} \bar{D}_{l}(s, \theta) G_{m}(s, \theta) \bar{D}_{r}(s, \theta)
\end{aligned}
$$

Due to its diagonal structure, uncertainty and nominal delay commute, i.e $\Delta_{l(r)} \bar{D}_{l(r)}=\bar{D}_{l(r)} \Delta_{l(r)}$. Here, the nominal (delayed) model has been defined as $G_{o}(s, \theta)$ and the rational (delay-free) part as $G_{m}(s, \theta)$.

The uncertain model is obtained from the Smith Predictor structure (Figure 2) between input $u$ and the feedback output $f$ :

$$
\begin{aligned}
& {\left[G_{m}(s, \theta)+G(s, \theta)-G_{o}(s, \theta)\right]=} \\
& =\left[\Delta_{l}(s) G_{o}(s, \theta) \Delta_{r}(s)-G_{o}(s, \theta)+G_{m}(s, \theta)\right]
\end{aligned}
$$

A simple way to include delay uncertainty in the set of models is to cover it completely with global uncertainty that does not distinguish between the different input/output channels. In this case, we may rewrite Eq. (14) in the following way:

$$
\left[\Delta_{l}(s)-I\right] G_{o}(s, \theta)\left[\begin{array}{c}
\Delta_{r}(s) \\
I
\end{array}\right]+G_{m}(s, \theta) .
$$

Then, according to (Sanchez-Peña, 2009), the uncertainty in the delay can be covered by a dynamic uncertain set of models as in Eq. (9). 


\section{LPV CONTROL OF THE PLANT}

\section{A. LPV General Framework}

The LPV plant (6) after the application of the factorization (10) and the MIMO Smith Predictor scheme (Figure 2), can be described by state-space equations of the form

$$
\begin{gathered}
\dot{x}(t)=A(\theta) x(t)+B(\theta) u(t)+B_{w}(\theta) w(t) \\
z(t)=C_{z}(\theta) x(t)+D_{z u}(\theta) u(t)+D_{z w}(\theta) w(t) \\
q(t)=C_{q}(\theta) x(t)+D_{q u}(\theta) u(t)+D_{q w}(\theta) w(t)
\end{gathered}
$$

where $x \in \mathfrak{R}^{\mathrm{n}}$ is the state vector, $u \in \mathfrak{R}^{\mathrm{m} 1}$ and $w \in \mathfrak{R}^{\mathrm{m} 2}$ are the control and disturbance input vectors, respectively, $z \in \mathfrak{R}^{\mathrm{p} 1}$ and $q \in \mathfrak{R}^{\mathrm{p} 2}$ are the measured and controlled output vectors, respectively. $A(\cdot), B(\cdot)$, $B_{w}(\cdot), C_{z}(\cdot), C_{q}(\cdot), D_{\mathrm{qu}}(\cdot), D_{z u}(\cdot), D_{z w}(\cdot), D_{q w}(\cdot)$ are continuous matrix valued functions of the time varying parameter vector $\theta(t) \in \Theta \subset \mathfrak{R}^{1}, \Theta$ being a polytope with $r$ vertices. Performance is defined as requiring a bounded output $q(t)$ for any bounded external signal $w(t)$, both measured by their energy integral. The synthesis technique for LPV systems is based on the following results:

Theorem 1. (Quadratic $H_{\infty}$ Performance) (see (Apkarian, 1995a)). The LPV system given by Eq.(16) is QS and has quadratic $H_{\infty}$ performance if there exists a positive definite matrix $X>0$ such that

$$
B_{[A(\theta), B(\theta), C(\theta), D(\theta)]}^{0}(X, \gamma):=\left[\begin{array}{ccc}
A^{T}(\theta) X+X A(\theta) & X B(\theta) & C^{T}(\theta) \\
B^{T}(\theta) X & -\gamma I & D^{T}(\theta) \\
C(\theta) & D(\theta) & -\gamma I
\end{array}\right]<0
$$

for all admissible values of the parameter $\theta$.

Remark 1. According (Apkarian, 1995a), the previous problem should fulfill the following 
assumptions:

1) $D_{q u}(\theta)=0$ or equivalently $D_{q u_{i}}=0$ for $i=1,2, . ., r$.

2) $B(\theta), C_{q}(\theta), D_{z u}(\theta), D_{q w}(\theta)$ are parameter-independent

3) $B_{i}=B, C_{q_{i}}=C, D_{z u_{i}}=D_{z u}, D_{q w_{i}}=D_{q w}$ for $i=1,2, \ldots, r$.

The pairs $[A(\theta), B]$ and $\left[A(\theta), C_{\mathrm{q}}\right]$ are quadratically stabilizable and detectable over $\Theta$, respectively.

Theorem 2. (Vertex Property) (see (Apkarian, 1995a)). Consider a polytopic linear parameter-varying plant as in Eq. (16), where

$$
\left[\begin{array}{ll}
A(\theta) & B(\theta) \\
C(\theta) & D(\theta)
\end{array}\right] \in \Theta:=C o\left\{\left[\begin{array}{cc}
A_{i} & B_{i} \\
C_{i} & D_{i}
\end{array}\right], \quad i=1, \ldots, r\right\}
$$

and assume $A, B, C, D$ are affine functions of $\theta$, then the following items are equivalent:

(i) The system is quadratic stable with Quadratic $H_{\infty}$ performance $\gamma$.

(ii) There exists a positive definite matrix $X>0$, which satisfies the following LMIs:

$$
B_{\left[A_{i}, B_{i}, C_{i}, D_{i}\right]}^{0}(X, \gamma)<0, \quad i=1,2, \ldots, r
$$

In this case, Theorem 1 should only be verified on the vertices of the parameter polytope $\Theta$, which in turn implies that the optimization problem reduces to a finite number of LMIs, which makes such an approach appealing.

\section{B. LPV Gain Scheduling Control Methodology}

The control design specifications that will be considered are a mixture of performance and robustness objectives arranged as a mixed sensitivity problem (MSP) (Skogestad, 1997),

$$
\left\|\left[\begin{array}{lll}
W_{e} S & W_{u} K S & W_{\Delta} T
\end{array}\right]\right\|_{\infty}^{T}<\gamma \leq 1
$$


which is illustrated in Figure 3. Here, $S$ is the sensitivity function and $T$ its complement. The transfer functions in Eq. (19) represent weighted tracking error (or disturbance rejection), weighted control action and robust stability, respectively. In order to limit the control energy and bandwidth of the controller, a weight $W_{u}$ is included in the design. Such weight is a transfer function with a crossover frequency approximately equal to that of the desired closed-loop bandwidth. The weight for the complementary sensitivity, $W_{\Delta}$, captures the uncertainty of the plant model (in this case coming from the delay measurement error) and also limits the closed loop bandwidth. Typically, a disturbance in the system output is a low frequency signal, and therefore it will be successfully rejected if the minimum value of $S$ is achieved over the same frequency band. This is performed by selecting a weight $W_{e}$, with a bandwidth equal to that of the disturbance in the controller design specifications. Performance is a combination of weighted error and control action minimization measured in terms of the energy integrals of the input and output signals involved. This control design problem will be solved using the concepts in Section III and Section IV.A, and using the fact that the time varying parameters enter affinely in the augmented model and are approximated by a polytopic region.

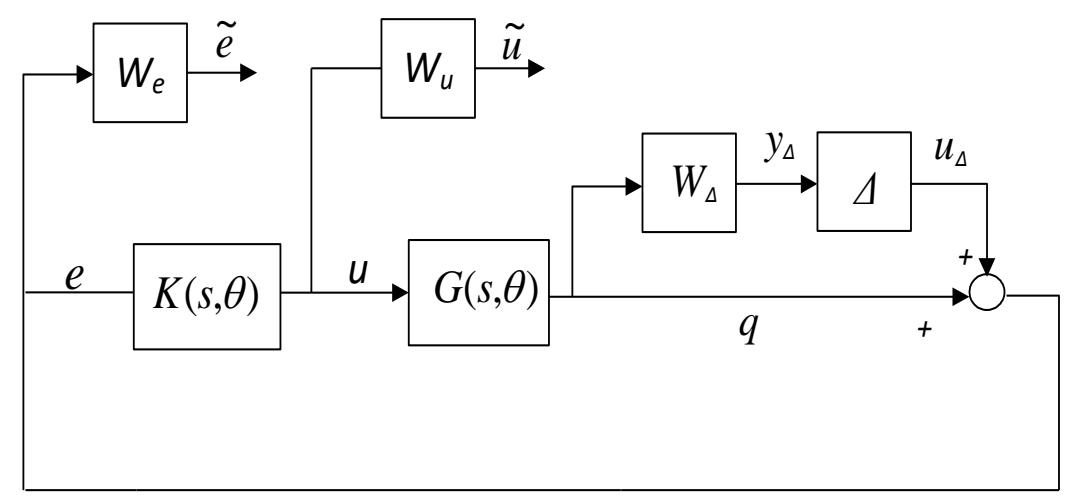

Figure 3. Proposed LPV feedback system scheme (MSP scheme).

A scheduling static time varying state feedback controller which satisfies QS and performance specifications is designed. This controller also guarantees "frozen" closed loop pole locations inside a desired LMI region. 
Since the LPV plant is represented in polytopic form, the controller $K(\theta)$ is designed according to:

$$
K(\theta) \in \operatorname{Co}\left\{K\left(\theta_{1}\right), K\left(\theta_{2}\right), \ldots, K\left(\theta_{r}\right)\right\}=\left\{\sum_{i=1}^{r} \lambda_{i} K_{i}: K_{i}=K\left(\theta_{i}\right), i=1, \ldots, r\right\}
$$

where: $C o$ is the convex hull of the polytope of vertex controllers, $\theta_{i}, i=1, \ldots, r$ are the vertices of the parameter polytope $\Theta$ and the polytopic coordinates $\lambda_{i} \geq 0, i=1, \ldots, r$ satisfy $\theta=\sum_{i=1}^{r} \lambda_{i} \theta_{r}$ with $\sum_{i=1}^{r} \lambda_{i}=1$.

Finally, the closed-loop system is $\dot{x}_{c l}=A_{c l}(\theta) x_{c l}+B_{c l} w$.

\section{CONTROL OF A TWO-POOL CANAL SYSTEM}

The test bench canal used to illustrate the proposed approach presented in this work consists of a multiple reach canal with two pools equipped with two sluices gates and a downstream spillway (see Figure 4). A servomotor is used in each gate position $\left(u_{1}\right.$ and $\left.u_{2}\right)$ and there are two level sensors located at the end of the two canal pools $\left(y_{d}{ }^{1}\right.$ and $\left.y_{d}{ }^{2}\right)$. Upstream of the first gate there is a reservoir with a constant level $H=3.5 \mathrm{~m}$. The total length of the first pool is $L_{1}=2 \mathrm{~km}$ while in the in the second $L_{2}=$ $4 \mathrm{~km}$. The canal width, bottom slope and Manning roughness coefficient are $B=2.5 \mathrm{~m}, I_{0}=5.10^{-4}$ and $n$ $=0.014$. The operating range of the gate is limited to the interval $U \in[0,0.9] \mathrm{m}$, the gate discharge coefficient and gate width are $C_{d g}=0.6$ and $b=2.5 \mathrm{~m}$. Finally, the downstream spillway height and coefficient are $Y_{s}=0.7 \mathrm{~m}$ and $C_{d s}=2.66$, respectively.

The verification of the methodology proposed in this work is carried out by a high-fidelity irrigation canal simulator, which represents water dynamics by Saint-Venant equations of an accurate and complete way. This simulator has been developed by the "Modeling and Control of Water Systems" group at the Automatic Control Department, UPC (Bolea and Blesa, 2000).

The way to represent the MIMO control model for two pool canal system is:

$$
\left[\begin{array}{c}
Y_{d i} \\
Y_{d i+1}
\end{array}\right]=\left[\begin{array}{ll}
P_{11}(s) & P_{12}(s) \\
P_{21}(s) & P_{22}(s)
\end{array}\right]\left[\begin{array}{c}
U_{i}(s) \\
U_{i+1}(s)
\end{array}\right]
$$


where $P_{11}, P_{12}, P_{21}$ and $P_{22}$ are the transfer functions that related the input vector (gate opening of each gate) with the output vector (downstream level in each pool) (Bolea, 2014).

$$
\begin{aligned}
& P_{11}(s)=\frac{K_{11}\left(\theta_{1}\right)}{T_{11}\left(\theta_{2}\right) s+1} e^{-\tau_{11}\left(\theta_{1}\right) s}, P_{11}(s)=\frac{K_{11}\left(\theta_{1}\right)}{T_{11}\left(\theta_{2}\right) s+1} e^{-\tau_{11}\left(\theta_{1}\right) s}, \\
& P_{11}(s)=\frac{K_{11}\left(\theta_{1}\right)}{T_{11}\left(\theta_{2}\right) s+1} e^{-\tau_{11}\left(\theta_{1}\right) s}, P_{11}(s)=\frac{K_{11}\left(\theta_{1}\right)}{T_{11}\left(\theta_{2}\right) s+1} e^{-\tau_{11}\left(\theta_{1}\right) s}
\end{aligned}
$$

where the gains, the time constants and the delays of each transfer function are the linear varying parameters of the model because they vary according to two gain scheduling variables $\left(u_{1}, u_{2}\right)$, that represent the opening gates of the pool 1 and pool 2, respectively. The model structure is given by LPV first order plus delay time (FOPDT) transfer functions Eq. (22). In particular, for the proposed two-pool system (Figure 4), if $t_{1}$ is the travelling time of the water to cover the first pool of length $L_{1}$ and $t_{2}$ is the travelling time of the water to cover the second pool of length $L_{2}$, the different delays associated to each input/output channel are: $\tau_{11}=t_{1}, \tau_{12}=0, \tau_{21}=t_{1}+t_{2}, \tau_{22}=t_{2}$. This means that the relation between delays established in Eq. (11) holds.

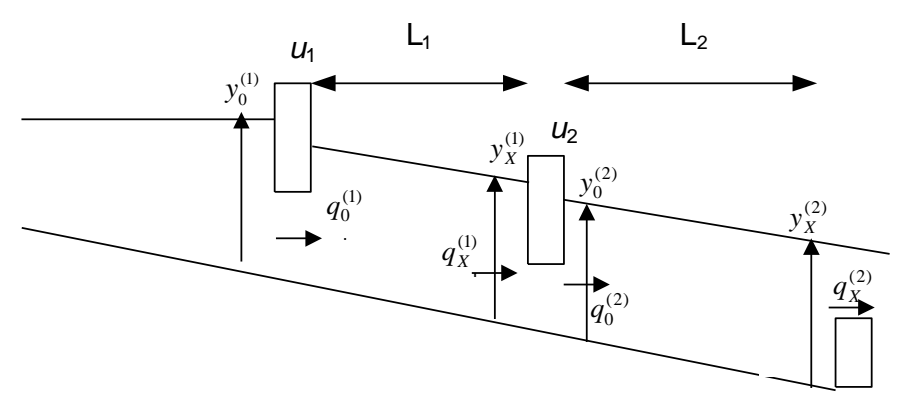

Figure 4. Two-pool canal system.

\section{A. Identification of the canal LPV model}

In order to apply the control design approach presented in this paper, first the model presented in (20) should be estimated using data. In the literature, there exist two main approaches for the identification 
of LPV models: a global (see e.g. (Bamieh, 2002) and a local (De Caigny, 2009) one. The global approach is based on the assumption that it is possible to perform a global identification experiment by exciting the system while the scheduling parameters are persistently changing the system dynamics. This assumption, however, may be difficult to satisfy in many cases. In the case that it is impossible to perform a global experiment, it is appropriate to use the local LPV identification approach, based on the interpolation of a set of local LTI models that are estimated using a set of local measurements, obtained by exciting the system at different fixed operating conditions, that is, for constant values of the scheduling parameters. To estimate the parameters of the LPV model(22), a methodology inspired in the one proposed (De Caigny, 2009) is used as described in detail in (Bolea et al., 2014b).

The parameters of the LPV model in Eq.(23) are estimated experimentally by applying a set of steps to each input $\left(u_{1}, u_{2}\right)$ that sweeps all the operating points. Each linear varying parameter depends on the gain scheduling variables (gate openings) $\theta=\left(\theta_{1}, \theta_{2}\right)=\left(u_{1}, u_{2}\right)$ as indicated in Eq.(23). The identification of the dynamics is carried out using the LPV identification approach presented in this section, based on performing LTI identifications at several operating points. It is assumed that the variation of the parameters $(K, T, \tau)$ with the scheduling variable $\theta$ can be approximated by a polynomial as follows :

$$
\begin{gathered}
K_{\mathrm{ij}}\left(u_{\mathrm{j}}\right)=a_{\mathrm{ij} 2} u_{\mathrm{j}}^{2}+a_{\mathrm{ij} 1} u_{\mathrm{j}}+a_{\mathrm{ij} 0} ; \\
T_{\mathrm{ij}}\left(u_{\mathrm{j}}\right)=b_{\mathrm{ij} 2} u_{\mathrm{j}}^{2}+b_{\mathrm{ij} 1} u_{\mathrm{j}}+b_{\mathrm{ij} 0} \\
\tau_{\mathrm{ij}}\left(u_{\mathrm{j}}\right)=c_{\mathrm{ij} 2} u_{1}^{2}+c_{\mathrm{ij} 1} u_{1}+c_{\mathrm{ij} 0}
\end{gathered}
$$

The values of the coefficients of the previous polynomials are shown in the Table I and have been obtained by interpolating the parameters at the different operating points. Then, a control model is developed which covers all the operation range, $u_{j} \in[0,0.9] j=1,2$, representing the suitable behaviour of the canal. The parameters of the system vary in these intervals presented in Table II with constant time and delay expressed in seconds. For more details in the identification procedure, see (Bolea et al., 2014b) 
TABLE I

COEFFICIENTS OF THE POLYNOMIALS OF EQ(26)

\begin{tabular}{|c|c|c|c|c|c|c|c|c|c|c|}
\hline & $a_{\mathrm{ij} 2}$ & $a_{\mathrm{ij} 1}$ & $a_{\mathrm{ij}}$ & $b_{\mathrm{ij} 2}$ & $b_{\mathrm{ij} 1}$ & $b_{\mathrm{ij} 0}$ & $c_{\mathrm{ij} 2}$ & $c_{\mathrm{ij} 1}$ & $c_{\mathrm{ij} 0}$ \\
\hline \multirow{2}{*}{ 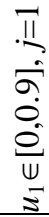 } & .II & 2.38 & -7.40 & 6.38 & 1308.45 & -1925.01 & 2222.66 & 881.42 & -1398.14 & 844.37 \\
\hline & II & 3.77 & -6.08 & 2.56 & -296.43 & 204.64 & 1855.92 & 580.47 & -1281.38 & 1883.06 \\
\hline \multirow{2}{*}{ 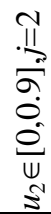 } & .II & 1.77 & 2.70 & -4.96 & 989.05 & -1502.23 & 2360.87 & 0 & 0 & 0 \\
\hline & II & 5.20 & -7.98 & 3.10 & -5.95 & -107.73 & 1521.78 & -550.77 & 362.99 & 959.47 \\
\hline
\end{tabular}

TABLE II

INTERVAL BOUNDING PARAMETER VARIATIONS

\begin{tabular}{|c|c|c|c|c|}
\hline Parameter & $K_{11}$ & $K_{21}$ & $K_{12}$ & $K_{22}$ \\
\hline Interval & {$[1.65,5.25]$} & {$[0.15,1.5]$} & {$[-4.35,-1.1]$} & {$[0.12,1.7]$} \\
\hline
\end{tabular}

\begin{tabular}{|c|c|c|c|c|}
\hline Parameter & $T_{11}$ & $T_{21}$ & $T_{12}$ & $T_{22}$ \\
\hline Interval & {$[1550,1920]$} & {$[1800,1900]$} & {$[1800,2100]$} & {$[1430,1500]$} \\
\hline
\end{tabular}

\begin{tabular}{|c|c|c|c|c|}
\hline Parameter & $\tau_{11}$ & $\tau_{21}$ & $\tau_{12}$ & $\tau_{22}$ \\
\hline Interval & {$[300,580]$} & {$[1200,1650]$} & 0 & {$[840,1010]$} \\
\hline
\end{tabular}

\section{B. LPV control design}

A LPV controller is designed following the method explained in Section IV. The LPV delay is compensated by a LPV SP, but there is an error in this estimation. This delay uncertainty (Eq.(14)) is covered with global dynamic uncertainty by the following weight (Figure 5, down) (Skogestad, 1997): 


$$
W_{\delta}(s)=\frac{16 s}{s+4 \cdot 10^{-3}}
$$

To test the improvement of the robust LPV control vs the robust LTI control (Sanchez-Peña, 2008), a comparative study is carried out. In the LTI case, the nominal delays and their uncertainty are as follows (time is expressed in minutes)(see Eq. (13)):

$$
D_{l}(s)=\left[\begin{array}{cc}
1 & 0 \\
0 & e^{-s\left(\bar{\tau}_{22} \pm 2\right)}
\end{array}\right], D_{r}(s)=\left[\begin{array}{cc}
e^{-s\left(\bar{\tau}_{11} \pm 2\right)} & 0 \\
0 & 1
\end{array}\right] \text { with } \delta= \pm 2 .
$$

The approach to select the LTI weight that covers this delay error is the same as in the LPV case. In the LPV case the weight is the following (Figure 5, top):

$$
W_{\delta}(s)=\frac{29 s}{s+2 \cdot 10^{-3}}
$$

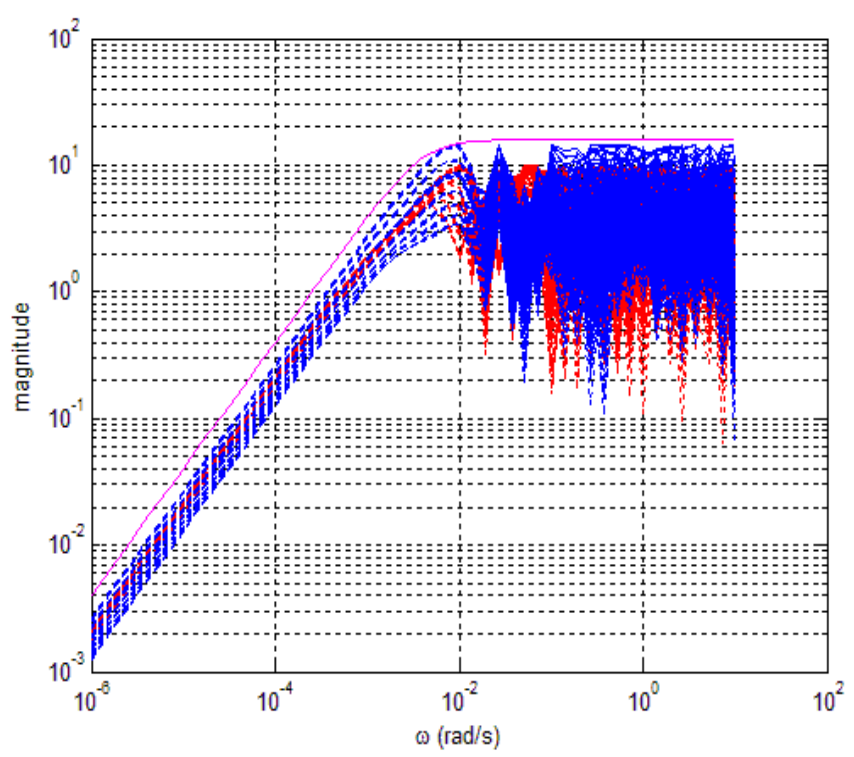




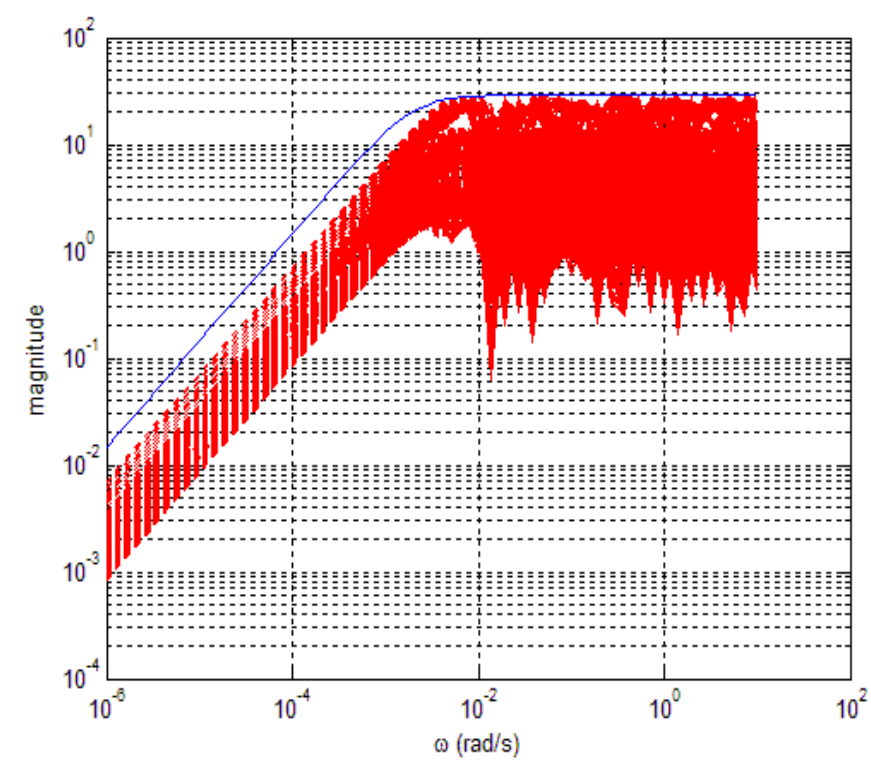

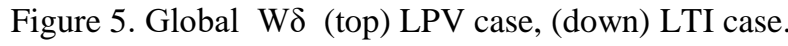

In Figure 6, the LPV vs LTI controllers are compared around one operation point: $u_{1} \in[0,0.3]$, $u_{2} \in[0.2,0.5]$. It can be observed that the LPV control is faster and with lower overshoot in both pools $\left(h_{1}\right.$ and $h_{2}$ ). Besides, the control signals of both gates are less aggressive in the LPV case and, as a consequence, the voltage consumption of both gates is lower than the consumption in the LTI case. In Figure 7 both controllers are simulated over all the operation range and the previous observations also apply. Clearly the LPV controller is more efficient in controlling the canal, which agrees with the theory. It can also be noticed that the degree of coupling of the second pool with respect to the first one is lower in the LPV controller case. 

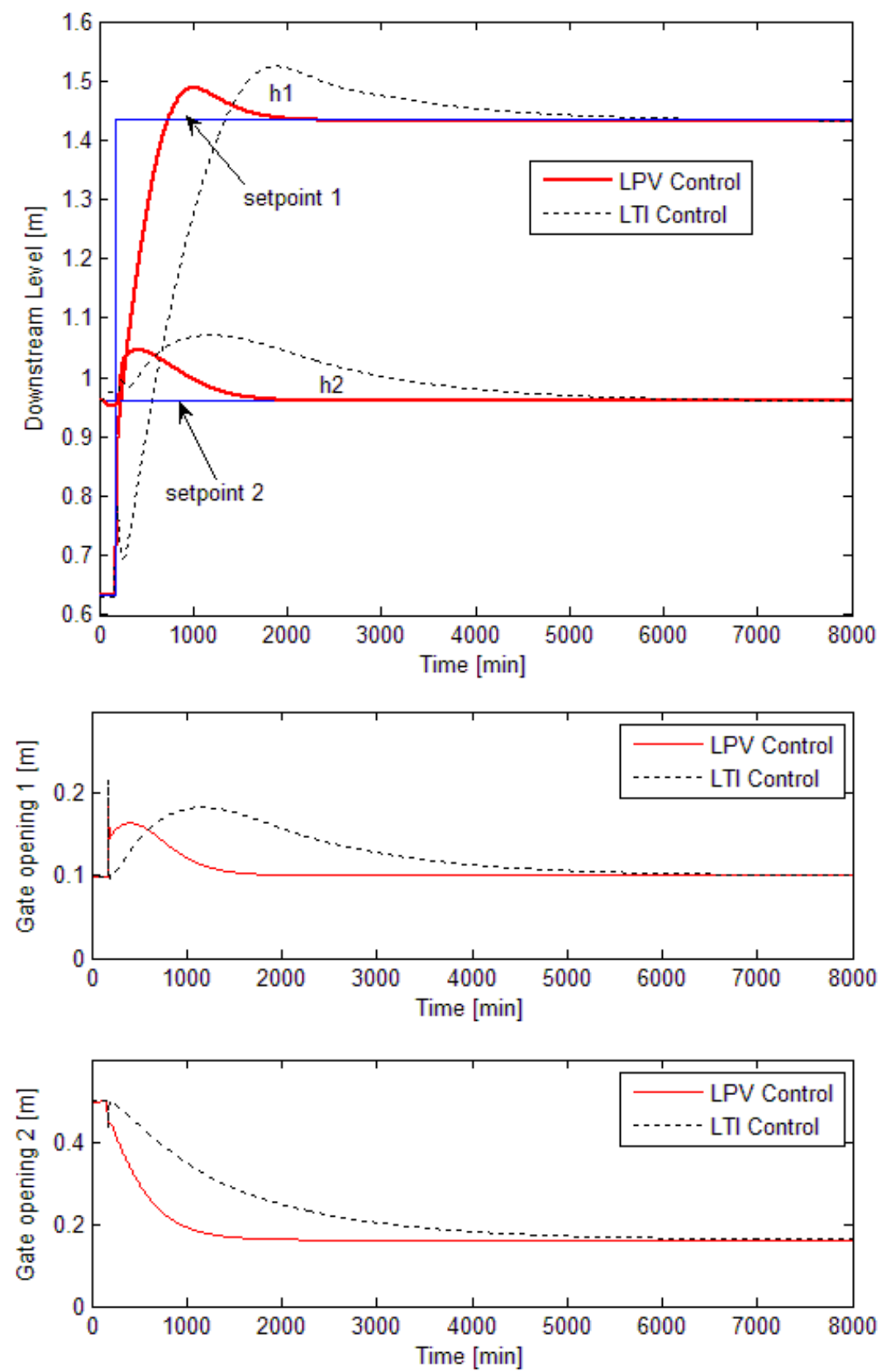

Figure 6. LPV and LTI control in the operation point $u_{1} \in[0,0.3], u_{2} \in[0.2,0.5]$. (Top) Desired and measured downstream levels of pool 1 and pool 2. (Down) Signal control of actuator of gate 1 and gate 2. 

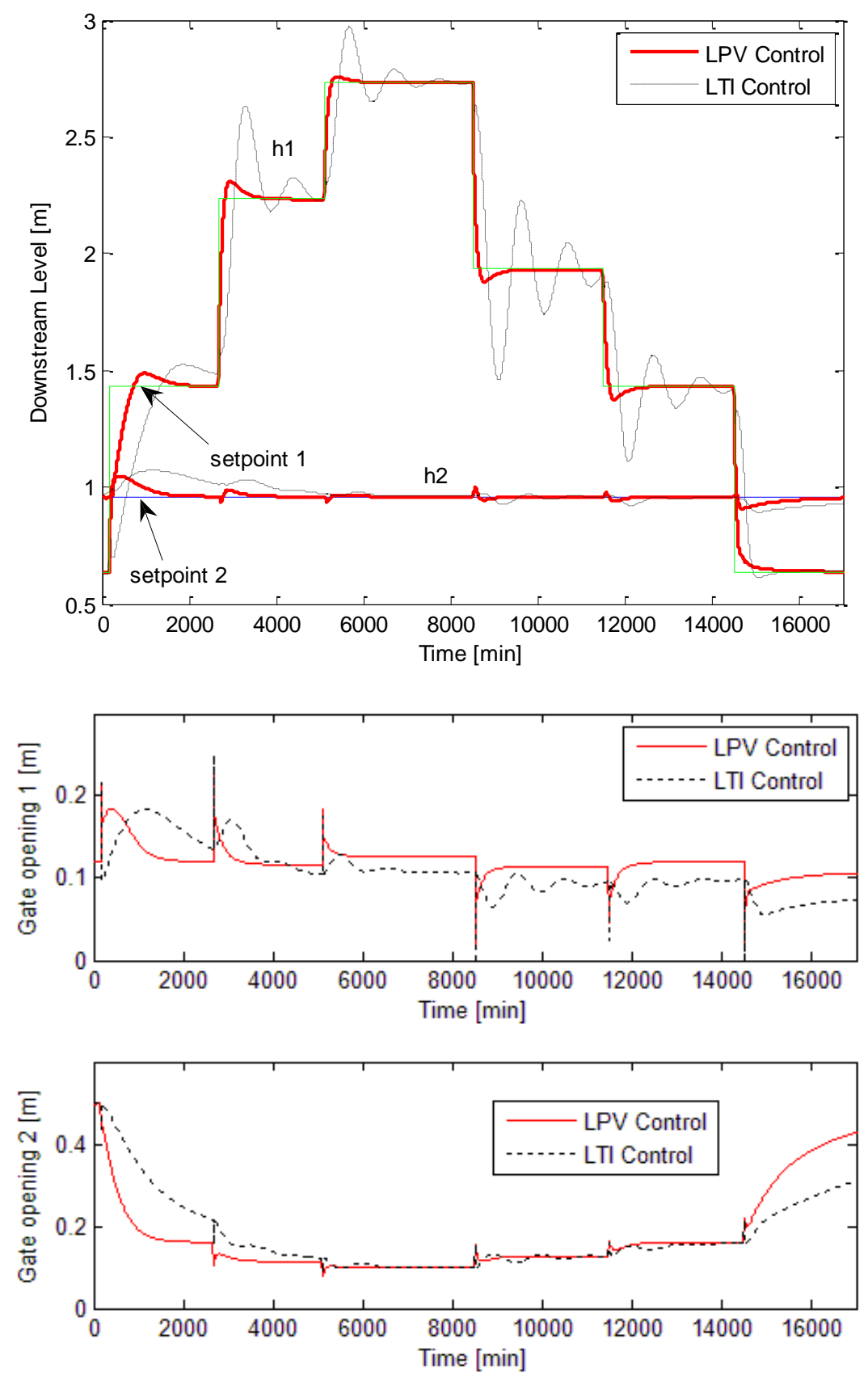

Figure 7. LPV and LTI control in a scenario (all operation range). (Top) Desired and measured downstream levels of pool 1 and pool 2. (Down) Signal control of actuator of gate 1 and gate 2.

\section{CONCLUSION}

In this paper is presented a new LPV robust controller with an LPV generalization of the extension of the classical Smith Predictor to MIMO systems with multiple uncertain delays presented in (SanchezPeña, Bolea and Puig, 2008). This novel methodology that presents a set of necessary and sufficient 
conditions under which a model can be separated into diagonal left and right pure delays and a rational matrix is. These conditions are satisfied by multiple pool open flow canal systems Therefore, a gainscheduling LPV MIMO SP controller based in the new method explained in this paper is used for controlling a multi-pool canal system. This LPV controller, compared with LTI control when applied to a two-pool canal system, shows very promising and satisfactory results.

\section{ACKNOWLEDGEMENTS}

This work has been funded by the Spanish Government (MINECO) through the project CICYT ECOCIS (ref. DPI2013-48243-C2-1-R) and by MINECO and FEDER through the project CICYT HARCRICS (ref. DPI2014-58104-R).

\section{REFERENCES}

Apkarian, P and Gahinet, P., "A convex characterization of gain-scheduled Ho controllers", IEEE Transactions on Automatic Control, vol.40, pp. 853-864. 1995 a.

Apkarian, P., Gahinet, P. and Becker, G., Self-Scheduled $H_{\infty}$ Control of Linear Parameter-Varying Systems: A Design Example, Automatica, vol.31,no.9, pp.1251-1261, 1995 b.

Apkarian, P and Tuan, H.D., "Parametrized LMIs in Control Theory", SIAM Journal on Control Optimization, vol.38, no.4, pp.1241-1264.

Bamieh, B. and Giarré, L., "Identification of linear parameter varying models", Int. J. Robust Nonlinear Control, 2002, vol.12, pp.841-853.

Barré de Saint Venant, A.J.C., "Théorie du Mouvement non-Permanent des Eaux avec Application aux Crues des Rivières et a l’Introduction des Marées dans Leurs Lits “, Comptes rendus, Acad. Sci., Paris, vol.73: pp.148-154, 237-240, 1871.

Becker, G. and Packard, A., "Robust performance of linear parametrically varying systems using parametrically-dependent linear feedback", System and Control Letters, vol.23, pp.205-215, 1994.

Belforte, G., Dabbene, F. and Gay, P., LPV Approximation of Distributed Parameter Systems in Environmental Modelling, Environmental Modelling \& Software, vol.2, 2002.

Biannic, J.-M., Apkarian, P., Missile Autopilot Design via a Modified LPV Synthesis Technique, Journal of Aerospace Science and Technology, vol.3, no.3, pp.153-160, 1999.

Bolea, Y. and Blesa, J., "Irrigation Canal Simulator (ICS)", Internal Report, Automatic Control Dept, Technical Univ. of Catalonia UPC, 2000.

Bolea, Y., Puig, V., and Blesa, J., "Gain-Scheduled Smith Predictor PID Based LPV Controller for Open-Flow Canal Control”, IEEE Transactions on Control Systems Technology, vol.22, no.2, 2014 a.

Bolea, Y., Puig, V. and Blesa, J., "Linear parameter varying modelling and identification for real-time control of open-flow irrigation canals”, Environmental Modelling \&Software, vol.53, pp. 87-97, 2014 b. 
Boudaoud, M., Gaudenzi De Faria, M., Le Gorrec, Y., Haddab, Y. and Lutz, P, “An output feedback LPV control strategy of a nonlinear electrostatic microgripper through a singular implicit modeling”, Control Engineering Practice, vol. 28, pp. 97-111, July 2014.

Castillo, F., Witrant, E., Prieur, C., Talon, V. and Dugard, L., "Fresh Air Fraction Control in Engines Using Dynamic Boundary Stabilization of LPV Hyperbolic Systems”, IEEE Trans. on Control Technology, vol.23(3), pp.963-974, 2015.

Chilali, M. and Gahinet, P., "H $\mathrm{H}_{\infty}$ design with pole placement constraints: An LMI approach", IEEE Trans. Automatic Control, vol.41(3), pp.358-367, 1996.

Chow, V.T., Open-channel hydraulics, N.Y:McGraw-Hill, 1959.

De Caigny, J., Camino, J.F., Swevers, J., Interpolating model identification for SISO linear parameter varying systems, Mech. Systems Signal Process, vol.23, pp.2305-2417, 2009.

Guoyan Cao, Karolos M. Grigoriadis, and Yaw D. Nyanteh, "LPV Control for the Full Region Operation of a Wind Turbine Integrated with Synchronous Generator", Mathematical Problems in Engineering, 15 pages, Volume 2015 (2015), Article ID 638120.

Hwanyub Joo and Sung Hyun Kim, "H $\mathrm{H}_{\infty}$ LPV Control with Pole Placement Constraints for Synchronous Buck Converters with Piecewise-Constant Loads", Mathematical Problems in Engineering, 8 pages, Volume 2015 (2015), Article ID 686857.

Leith, D. and Leithead, W. "Survey of gain-scheduling analysis and design”, International Journal of Control, vol.73 (2000), pp.1001-1025.

Liang Dong, Wen Cheng Tang and Da Fei Bao, "Interpolating gain-scheduled $\mathrm{H}_{\infty}$ loop shaping design for high speed ball screw feed drives", ISA Transactions, vol. 55, pp. 219-226, March 2015.

Litrico, X., and Fromion, V., "Simplified modeling of irrigation canals for controller design, Journal of Irrigation and Drainage Engineering, Vol. 130, No. 5, pp. 373-383, 2004

Litrico, X., and Fromion, V., "H $\mathrm{H}_{\infty}$ control of an irrigation canal pool with a mixed control politics", IEEE Trans. on Control Systems Technology, vol.14(1), pp. 99-111, 2006.

Ljung, L., System Identification Toolbox. For use with Matlab, MathWorks Inc., 1995.

Masubuchi I. and Kurata, I., “Gain-scheduled control via filtered scheduling parameters”, Automatica, vol. 47(8), pp. 18211826, August 2011.

Packard, A., “Gain-Scheduling via Linear Fractional Transformations”, Systems and Control Letters, vol.22, pp.79-92, 1994.

Packard, A. and Kantner, M., "Gain Scheduling the LPV Way", Proc. of the $35^{\text {th }}$ Conference on Decision and Control, Japan, Dec. 1995.

Rugh, W. and J. Shamma, "Research on gain scheduling”, Automatica, vol.36, pp.1401-1425, 2000.

Shamma, J.S. and Athans, M., "Guaranteed Properties of Gain Scheduled Control of Linear Parameter-varying Plants", Automatica, 27(3), pp.898-907, 1991.

Sánchez-Peña, R., Bolea, Y., and Puig, V., "MIMO Smith predictor: Global and structured robust performance analysis", Journal of Process Control, vol.19, no.1, pp.163-177, 2009.

Sato, M. and Peaucelle, D., "Gain-scheduled output-feedback controllers using inexact scheduling parameters for continuoustime LPV systems”, Automatica, vol.49(4), pp. 1019-1025, April 2013. 
Skogestad, S., and Postlethwaite, I., Multivariable feedback control. Analysis and Design, John Wiley \& Sons, 1997.

Wang, Y., Bevly, D.M. and Rajamani, R., "Interval observer design for LPV systems with parametric uncertainty", Automatica, vol. 60, pp.79-85, October 2015.

White, A., Zhen Ren, Guoming Zhu and Jongeun Choi, "Mixed H2/Hœ Observer-Based LPV Control of a Hydraulic Engine Cam Phasing Actuator", IEEE Trans. on Control Technology, vol.21(1), pp.229-238, 2013. 